\title{
Comparison between clinical significance of height-adjusted and weight-adjusted appendicular skeletal muscle mass
}

\author{
Taishi Furushima', Motohiko Miyachi ${ }^{2}$, Motoyuki lemitsu', Haruka Murakami ${ }^{2}$, Hiroshi Kawano ${ }^{3}$, Yuko Gando ${ }^{2}$, \\ Ryoko Kawakami ${ }^{4}$ and Kiyoshi Sanada ${ }^{1 *}$
}

\begin{abstract}
Background: This study aimed to compare relationships between height- or weight-adjusted appendicular skeletal muscle mass (ASM/Ht ${ }^{2}$ or ASM/Wt) and risk factors for cardiometabolic diseases or osteoporosis in Japanese men and women.

Methods: Subjects were healthy Japanese men $(n=583)$ and women $(n=1218)$. The study population included a young group (310 men and 357 women; age, 18-40 years) and a middle-aged and elderly group (273 men and 861 women; age, $\geq 41$ years). ASM was measured by dual-energy X-ray absorptiometry. The reference values for class 1 and 2 sarcopenia in each sex were defined as values one and two standard deviations below the sex-specific means of the young group, respectively.

Results: The reference values for class 1 and 2 sarcopenia defined by ASM/Ht ${ }^{2}$ were 7.77 and $6.89 \mathrm{~kg} / \mathrm{m}^{2}$ in men and 6 . 06 and $5.31 \mathrm{~kg} / \mathrm{m}^{2}$ in women, respectively. The reference values for ASM/Nt were 35.0 and $32.0 \%$ in men and 29.6 and $26.4 \%$ in women, respectively. In both men and women, ASM/Wt was negatively correlated with higher triglycerides (TG) and positively correlated with serum high-density lipoprotein cholesterol (HDL-C), but these associations were not found in height-adjusted ASM. In women, TG, systolic blood pressure, and diastolic blood pressure in sarcopenia defined by ASM/Wt were significantly higher than those in normal subjects, but these associations were not found in sarcopenia defined by $\mathrm{ASM} / \mathrm{Ht}^{2}$. Whole-body and regional bone mineral density in sarcopenia defined by $\mathrm{ASM} / \mathrm{Ht}^{2}$ were significantly lower than those in normal subjects, but these associations were not found in sarcopenia defined by ASM/Wt.
\end{abstract}

Conclusions: Weight-adjusted definition was able to identify cardiometabolic risk factors such as TG and HDL-C while height-adjusted definition could identify factors for osteoporosis.

Keywords: Sarcopenia, Definition, Body height, Body weight, Cardiometabolic diseases, Osteoporosis

\section{Background}

"Sarcopenia" refers to a reduction in muscle mass and function with age [1] and leads to physical disabilities [2, 3], falls [2], and osteoporosis [4]. Sarcopenia also increases the risk of developing chronic disorders, including cardiovascular diseases (CVD) [5] and type 2 diabetes [6].

\footnotetext{
* Correspondence: ksanada@fc.ritsumei.ac.jp

${ }^{1}$ College of Sport and Health Science, Ritsumeikan University, 1-1-1 Noji

Higashi, Kusatsu, Shiga 525-8577, Japan

Full list of author information is available at the end of the article
}

Although sarcopenia is often defined by relative muscle mass, different methods for normalizing values, such as by adjusting for height (Ht) and body weight (Wt), have been widely reported. Baumgartner et al. previously defined sarcopenia by height-adjusted appendicular skeletal muscle mass $\left(\mathrm{ASM} / \mathrm{Ht}^{2}, \mathrm{~kg} / \mathrm{m}^{2}\right)$ in a study involving Hispanic and non-Hispanic white men and women [2]. They reported that sarcopenia was significantly associated with self-reported physical disability in both sexes, independently of ethnicity, age, morbidity, obesity, income, and hearth condition. In contrast, Janssen et al. defined sarcopenia by weight-adjusted ASM 
(ASM/Wt $\times 100, \%)$ in a study of non-Hispanic white people, non-Hispanic black people, and Mexican Americans [7]. They also reported that the age-related decrease of relative muscle mass is significantly and independently associated with functional impairment and disability, particularly in older women.

With regard to Asian populations, Hong et al. investigated the association of sarcopenia defined by heightadjusted ASM and sites of fragility fractures in Chinese elderly [8]. Logistic regression analyses in that study revealed that lower height-adjusted ASM was associated with an increased risk of hip fracture both in men and women. In elderly Korean people, height-adjusted ASM was positively correlated with bone mineral density (BMD), and a strong correlation was found between sarcopenia and osteoporosis with risk of bone fracture [9].

Park et al. reported that CVD prevalence was positively associated with sarcopenia defined by weight-adjusted ASM in men after adjusting for CVD risk factors, but no such association was found in women [10]. Similarly, Byeon et al. reported that in a $\geq 60$-year-old Korean study population, sarcopenic individuals defined by weight-adjusted ASM had a higher Framingham risk score than nonsarcopenic individuals in a non-obese group [11]. Moreover, in a Thai population, Pongchaiyakul et al. found that an urban environment is the strongest predictive factor for sarcopenia defined by weight-adjusted ASM, followed by high body mass index (BMI) and age [12]. These previous studies suggest that the relationship between sarcopenia and the risk of lifestyle-related diseases such as cardiometabolic diseases and osteoporosis depends on the method used to normalize muscle mass in Asian populations.

Lim et al. previously compared risk factors for metabolic syndrome in Korean individuals with sarcopenia defined by height-adjusted and weight-adjusted ASM [13]. In that study, however, the sex-specific young reference group used to determine cut-off points for sarcopenia had a small number of subjects.

Although Sanada et al. reported reference values for height-adjusted ASM in sarcopenia for young Japanese adult men and women [14], it is unclear whether different relationships would result between sarcopenia defined by height-adjusted ASM or weight-adjusted ASM and risk factors for cardiometabolic diseases or osteoporosis in Japanese populations.

Against this backdrop, this study aimed to compare relationships between height- or weight-adjusted ASM and risk factors for cardiometabolic diseases or osteoporosis in Japanese men and women.

\section{Methods}

\section{Subjects}

Subjects were healthy Japanese men $(n=583)$ and women $(n=1218)$ aged $18-90$ years. The study population included a young group ( 310 men and 357 women; age, 18-40 years) which served as a basis to define the reference values referred by Baumgartner et al. [2], and a middle-aged and elderly group (273 men and 861 women; age, $\geq 41$ years). The subjects were recruited from people participating in a Nutrition and Exercise Intervention Study (NEXIS, registered at ClinicalTrials.gov, Identifier: NCT00926744). We excluded men and women with obesity (BMI $\geq 25$ ), CVD, and stroke, as determined using a medical history questionnaire. They were sedentary or moderately active and participated in a swimming, stretching, and "healthy gymnastics" program; however, none of the subjects participated in other vigorous sports activities. The purpose, procedures, and risks of the study were explained to all participants prior to inclusion, and all subjects gave their written informed consent before enrolling in the study. The study was performed in accordance with the guidelines of the Declaration of Helsinki and was approved by the Human Research Committee of the National Institute of Health and Nutrition, Tokyo, Japan (KENEI14-02). Body weight and height were recorded, and BMI was calculated as weight $(\mathrm{kg})$ divided by the square of the height $(\mathrm{m})$. Waist circumference (WC) was measured at the uppermost border of the iliac crests.

\section{Analysis of blood samples}

Blood was drawn from subjects in the seated position. Fasting $(>12 \mathrm{~h})$ blood samples were collected by venipuncture in tubes with or without ethylene diamine tetraacetic acid (for plasma or serum). Blood samples were centrifuged at $1500 \mathrm{rpm}$ for $15 \mathrm{~min}$ and stored at $-20{ }^{\circ} \mathrm{C}$. Serum concentration of triglycerides (TG) was determined using commercial kits (Mitsubishi Chemical Medience, Tokyo, Japan). Serum high-density lipoprotein cholesterol (HDL-C) was measured by an enzymatic method (Mitsubishi Chemical Medience). Whole-blood glycohemoglobin A1c (HbA1c) was measured by an enzymatic method (glycohemoglobin A1c kit; Mitsubishi Chemical Medience).

\section{Analysis of arterial blood pressure at rest}

Systolic blood pressure (SBP) and diastolic blood pressure (DBP) were measured at rest using a vascular testing device (Colin Medical Technology, Tokyo, Japan). Chronic arterial blood pressure levels at rest were measured with the same device over the brachial and dorsalis pedis arteries. Recordings were made in triplicate with subjects in the supine position. Brachial-ankle pulse wave velocity (baPWV), which provides qualitatively similar information to that derived from central arterial stiffness, was measured by the volume plethysmographic method.

\section{Whole-body dual-energy X-ray absorptiometry}

Lean soft tissue mass and BMD were determined by whole-body dual-energy X-ray absorptiometry (DXA) 
(Hologic QDR-4500A scanner; Hologic, Waltham, MA). Whole-body lean soft tissue mass was divided into several regions, i.e., the arms, legs, and trunk. Body composition was determined by Hologic software version 11.2:3 for Windows (Hologic, Waltham, MA). The reference values $\left(\mathrm{ASM} / \mathrm{Ht}^{2}, \mathrm{ASM} / \mathrm{Wt} \times 100\right)$ for class 1 and class 2 sarcopenia in each sex were defined as values one and two standard deviations (SD) below the sex-specific means of the reference data for young adults aged 18-40 years, respectively.

\section{Measures of fitness}

Handgrip strength of the right upper limb was measured using a handheld dynamometer. In the standing position, with the arm straight by the side, the subjects gripped the dynamometer as hard as possible for $3 \mathrm{~s}$ without pressing the instrument against the body or bending at the elbow. Value was recorded as the average of two trials.

\section{Statistical analysis}

All measurements and calculated values are expressed as mean \pm SD. Partial correlations between height-adjusted ASM and weight-adjusted ASM were analyzed after adjusting for age and percent body fat. Differences between prevalence of sarcopenia defined by height-adjusted ASM and weight-adjusted ASM were assessed by the chisquare test. We compared mean values of physical characteristics, body composition, fitness, and risk factors for cardiometabolic diseases between normal, class 1 and class 2 sarcopenia by one-way ANCOVA after adjusting for age and percent body fat. Student's unpaired $t$ test was used to test differences among normal, class 1 and class 2 sarcopenic subjects. The alpha level for testing significance was set at $P<0.05$. All statistical analyses were performed using StatView version 5.0 for Window (SAS Institute, Cary, NC, USA).

\section{Results}

The physical characteristics of young group and middleaged and elderly group are shown in Table 1. Heightadjusted ASM in young men and women aged 18-40 years were $8.66 \pm 0.89 \mathrm{~kg} / \mathrm{m}^{2}$ and $6.80 \pm 0.75 \mathrm{~kg} / \mathrm{m}^{2}$, respectively. Weight-adjusted ASM in young men and women were $38.1 \pm 3.0 \%$ and $32.7 \pm 3.2 \%$, respectively. Therefore, in this study, the cut-off values for class 1 sarcopenia using heightadjusted ASM in men and women were set as 7.77 and $6.06 \mathrm{~kg} / \mathrm{m}^{2}$ and the cut-off values for class 2 sarcopenia were set as 6.89 and $5.31 \mathrm{~kg} / \mathrm{m}^{2}$, respectively. In addition, the cut-off values for class 1 sarcopenia using weightadjusted ASM in men and women were set as 35.0 and $29.6 \%$ and the cut-off values for class 2 sarcopenia were set as 32.0 and $26.4 \%$, respectively. In the middle-aged and elderly group aged 41-90 years, height-adjusted ASM in men and women were $8.05 \pm 0.68 \mathrm{~kg} / \mathrm{m}^{2}$ and $6.47 \pm$ $0.58 \mathrm{~kg} / \mathrm{m}^{2}$, respectively. Weight-adjusted ASM in men and women were $35.9 \pm 2.6 \%$ and $30.3 \pm 2.9 \%$, respectively.

Partial correlation coefficients adjusted for age are shown in Table 2. After adjusting for age, partial correlation coefficients between height-adjusted ASM and weight-adjusted ASM were 0.577 in men $(P<0.001)$ and 0.467 in women $(P<0.001)$, and $\mathrm{Wt}, \mathrm{BMI}$, and $\mathrm{WC}$ were positively correlated with height-adjusted ASM but negatively correlated with weight-adjusted ASM in men and women $(P<0.01)$. HbA1c, TG, SBP, and DBP were negatively, and HDL-C was positively, correlated with weight-adjusted ASM $(P<0.01)$, but not with heightadjusted ASM in women. In men, lumbar spine BMD was positively correlated with $\mathrm{ASM} / \mathrm{Ht}^{2}(P<0.05)$, but not with $\mathrm{ASM} / \mathrm{Wt}$. ASM $/ \mathrm{Ht}^{2}$ had higher correlation coefficients with whole-body, arm, lumber spine, and leg BMD than ASM/Wt in both men and women. In men and women, baPWV was negatively correlated with both $\mathrm{ASM} / \mathrm{Ht}^{2}$ and ASM/Wt $(P<0.05)$.

Table 1 Physical characteristics of young group and middle-aged and elderly group

\begin{tabular}{|c|c|c|c|c|}
\hline & \multicolumn{2}{|c|}{ Young group } & \multicolumn{2}{|c|}{ Middle-aged and elderly group } \\
\hline & Men & Women & Men & Women \\
\hline Number & 310 & 357 & 273 & 861 \\
\hline Age (years) & $29 \pm 7$ & $29 \pm 7$ & $60 \pm 12$ & $60 \pm 11$ \\
\hline Height (cm) & $173.2 \pm 5.6$ & $160.4 \pm 5.9$ & $168.7 \pm 6.3$ & $155.7 \pm 5.8$ \\
\hline Weight (kg) & $68.6 \pm 8.8$ & $53.9 \pm 7.2$ & $63.9 \pm 6.7$ & $52.0 \pm 5.8$ \\
\hline BMI $\left(\mathrm{kg} / \mathrm{m}^{2}\right)$ & $22.9 \pm 2.8$ & $20.9 \pm 2.5$ & $22.4 \pm 1.5$ & $21.4 \pm 1.9$ \\
\hline WC (cm) & $79.2 \pm 7.9$ & $73.0 \pm 8.4$ & $82.1 \pm 5.6$ & $79.6 \pm 7.2$ \\
\hline Percent body fat (\%) & $16.7 \pm 4.8$ & $24.4 \pm 5.7$ & $20.0 \pm 3.9$ & $28.0 \pm 5.2$ \\
\hline Handgrip strength (kg) & $44.5 \pm 7.1$ & $29.3 \pm 5.3$ & $38.6 \pm 7.3$ & $25.1 \pm 4.5$ \\
\hline $\mathrm{ASM} / \mathrm{Ht}^{2}\left(\mathrm{~kg} / \mathrm{m}^{2}\right)$ & $8.66 \pm 0.89$ & $6.80 \pm 0.75$ & $8.05 \pm 0.68$ & $6.47 \pm 0.58$ \\
\hline ASM/Wt $\times 100(\%)$ & $38.1 \pm 3.0$ & $32.7 \pm 3.2$ & $35.9 \pm 2.6$ & $30.3 \pm 2.9$ \\
\hline
\end{tabular}

Data are presented as mean $\pm \mathrm{SD}$

$B M I$ body mass index, WC waist circumference, $A S M$ appendicular skeletal muscle mass, $H t$ height, Wt weight 
Table 2 Partial correlation coefficients adjusted for age between ASM and measured values in middle-aged and elderly men and women

\begin{tabular}{|c|c|c|c|c|c|c|c|c|}
\hline & \multicolumn{4}{|l|}{ Men } & \multicolumn{4}{|c|}{ Women } \\
\hline & \multicolumn{2}{|c|}{$\mathrm{ASM} / \mathrm{Ht}^{2}\left(\mathrm{~kg} / \mathrm{m}^{2}\right)$} & \multicolumn{2}{|c|}{ ASM/Wt× $100(\%)$} & \multicolumn{2}{|c|}{$\mathrm{ASM} / \mathrm{Ht}^{2}\left(\mathrm{~kg} / \mathrm{m}^{2}\right)$} & \multicolumn{2}{|c|}{ ASM/Wt× $100(\%)$} \\
\hline & $R$ & $P$ value & $\bar{R}$ & $P$ value & $\bar{R}$ & $P$ value & $\bar{R}$ & $P$ value \\
\hline $\mathrm{ASM} / \mathrm{Ht}^{2}\left(\mathrm{~kg} / \mathrm{m}^{2}\right)$ & - & - & 0.577 & $<0.001$ & - & - & 0.467 & $<0.001$ \\
\hline ASM/Wt× $100(\%)$ & 0.577 & $<0.001$ & - & - & 0.467 & $<0.001$ & - & - \\
\hline Height (cm) & 0.086 & N.S. & 0.097 & $<0.01$ & 0.039 & N.S. & 0.148 & $<0.001$ \\
\hline Weight (kg) & 0.427 & $<0.001$ & -0.199 & $<0.01$ & 0.431 & $<0.001$ & -0.373 & $<0.001$ \\
\hline BMI $\left(\mathrm{kg} / \mathrm{m}^{2}\right)$ & 0.538 & $<0.001$ & -0.374 & $<0.001$ & 0.478 & $<0.001$ & -0.549 & $<0.001$ \\
\hline WC (cm) & 0.198 & $<0.01$ & -0.485 & $<0.001$ & 0.209 & $<0.001$ & -0.558 & $<0.001$ \\
\hline Percent body fat (\%) & -0.239 & $<0.001$ & -0.729 & $<0.001$ & -0.251 & $<0.001$ & -0.896 & $<0.001$ \\
\hline Handgrip strength (kg) & 0.383 & $<0.001$ & 0.394 & $<0.001$ & 0.340 & $<0.001$ & 0.283 & $<0.001$ \\
\hline Whole-body BMD $\left(\mathrm{g} / \mathrm{cm}^{2}\right)$ & 0.352 & $<0.001$ & 0.139 & $<0.05$ & 0.275 & $<0.001$ & 0.207 & $<0.001$ \\
\hline Arm BMD $\left(\mathrm{g} / \mathrm{cm}^{2}\right)$ & 0.422 & $<0.001$ & 0.190 & $<0.01$ & 0.324 & $<0.001$ & 0.249 & $<0.001$ \\
\hline Lumbar spine BMD $\left(\mathrm{g} / \mathrm{cm}^{2}\right)$ & 0.206 & $<0.05$ & 0.080 & N.S. & 0.244 & $<0.001$ & 0.150 & $<0.001$ \\
\hline Leg BMD $\left(\mathrm{g} / \mathrm{cm}^{2}\right)$ & 0.442 & $<0.001$ & 0.167 & $<0.01$ & 0.378 & $<0.001$ & 0.174 & $<0.001$ \\
\hline HbA1c (\%) & -0.032 & N.S. & 0.019 & N.S. & 0.009 & N.S. & -0.104 & $<0.01$ \\
\hline TG (mg/dl) & -0.032 & N.S. & -0.153 & $<0.05$ & -0.045 & N.S. & -0.224 & $<0.001$ \\
\hline $\mathrm{HDL}-\mathrm{C}(\mathrm{mg} / \mathrm{dl})$ & 0.051 & N.S. & 0.246 & $<0.001$ & 0.001 & N.S. & 0.333 & $<0.001$ \\
\hline TG/HDL ratio & -0.033 & N.S. & -0.179 & $<0.05$ & -0.039 & N.S. & -0.268 & $<0.001$ \\
\hline SBP $(\mathrm{mmHg})$ & 0.060 & N.S. & -0.011 & N.S. & 0.035 & N.S. & -0.114 & $<0.01$ \\
\hline $\mathrm{DBP}(\mathrm{mmHg})$ & 0.141 & $<0.05$ & -0.039 & N.S. & 0.034 & N.S. & -0.127 & $<0.001$ \\
\hline baPWV (cm/s) & -0.214 & $<0.01$ & -0.149 & $<0.05$ & -0.153 & $<0.001$ & -0.141 & $<0.001$ \\
\hline
\end{tabular}

ASM appendicular skeletal muscle mass, $H t$ height, Wt weight, BMI body mass index, WC waist circumference, BMD bone mineral density, $H b A 1 C$ glycohemoglobin A1c, $T G$ triglycerides, $H D L-C$ high-density lipoprotein cholesterol, SBP systolic blood pressure, $D B P$ diastolic blood pressure, baPWV brachial-ankle pulse wave velocity

Partial correlation coefficients adjusted for age and percent body fat are shown in Table 3. After adjusting for age and percent body fat, partial correlation coefficients between height-adjusted ASM and weightadjusted ASM were 0.630 in men $(P<0.001)$ and 0.577 in women $(P<0.001)$. HbA1c, TG, and TG/HDL-C were not significantly correlated with $\mathrm{ASM} / \mathrm{Ht}^{2}$ and ASM/Wt in both men and women. In men, whole-body, arm, lumbar spine, and leg BMD were positively correlated with $\mathrm{ASM} / \mathrm{Ht}^{2} \quad(P<0.05)$, but not with ASM/Wt. In women, whole-body and lumbar spine BMD were positively correlated with $\mathrm{ASM} / \mathrm{Ht}^{2}(P<0.001)$, but not with ASM/Wt. In men and women, baPWV was negatively correlated with both ASM/ $\mathrm{Ht}^{2}$ and ASM/Wt $(P<0.001)$.

When sarcopenia was defined by weight-adjusted ASM, the prevalence rates of class 1 and class 2 sarcopenia in the subjects aged $41-90$ years were $32.6 \%$ (89/ $273)$ and $5.9 \%(16 / 273)$ in men (Table 4$)$ and $35.5 \%$ $(306 / 861)$ and $7.1 \%(61 / 861)$ in women (Table 5), respectively. When defined by height-adjusted ASM, the prevalence rates of class 1 and class 2 sarcopenia in the subjects aged $41-90$ years were $27.8 \%(76 / 273)$ and $4.4 \%$ $(12 / 273)$ in men (Table 4$)$ and $22.3 \%(192 / 861)$ and $1.5 \%$ $(13 / 861)$ in women (Table 5), respectively. The prevalence rates of sarcopenia significantly differed when defined by height-adjusted ASM and weight-adjusted ASM in both men and women $(P<0.001$; chi-square test). In the subjects aged 65-90 years, the prevalence rates of class 1 and class 2 sarcopenia defined by weight-adjusted ASM were $39.8 \%(43 / 108)$ and $10.2 \%(11 / 108)$ in men, and $39.8 \%$ $(125 / 314)$ and $9.2 \%(29 / 314)$ in women, respectively. When defined by height-adjusted ASM, the prevalence rates of class 1 and class 2 sarcopenia were 39.8\% (43/108) and $9.3 \%(10 / 108)$ in men and $24.2 \%(76 / 314)$ and $2.5 \%$ $(8 / 314)$ in women, respectively.

Health-related indexes of middle-aged and elderly men (age $\geq 41$ years) are shown in Table 4 . When sarcopenia was defined by height-adjusted ASM, BMI and WC in class 1 and class 2 sarcopenic subjects were significantly lower than those in normal subjects $(P<0.05)$, but when sarcopenia was defined by weightadjusted ASM, BMI and WC did not significantly differ between the three groups. Whole-body BMD, arm $\mathrm{BMD}$, lumbar spine BMD, and leg BMD in class 1 and 2 sarcopenic subjects defined by height-adjusted ASM were significantly lower than those in normal subjects $(P<0.05)$. When sarcopenia was defined by weightadjusted ASM, those in class 1 and 2 sarcopenic 
Table 3 Partial correlation coefficients adjusted for age and percent body fat between ASM and measured values in middle-aged and elderly men and women

\begin{tabular}{|c|c|c|c|c|c|c|c|c|}
\hline & \multicolumn{4}{|l|}{ Men } & \multicolumn{4}{|c|}{ Women } \\
\hline & \multicolumn{2}{|c|}{$\mathrm{ASM} / \mathrm{Ht}^{2}\left(\mathrm{~kg} / \mathrm{m}^{2}\right)$} & \multicolumn{2}{|c|}{ ASM/Wt× $100(\%)$} & \multicolumn{2}{|c|}{$\mathrm{ASM} / \mathrm{Ht}^{2}\left(\mathrm{~kg} / \mathrm{m}^{2}\right)$} & \multicolumn{2}{|c|}{ ASM/Wt× $100(\%)$} \\
\hline & $R$ & $P$ value & $R$ & $P$ value & $R$ & $P$ value & $R$ & $P$ value \\
\hline $\mathrm{ASM} / \mathrm{Ht}^{2}\left(\mathrm{~kg} / \mathrm{m}^{2}\right)$ & - & - & 0.630 & $<0.001$ & - & - & 0.577 & $<0.001$ \\
\hline ASM/Wt× $100(\%)$ & 0.630 & $<0.001$ & - & - & 0.577 & $<0.001$ & - & - \\
\hline Height (cm) & 0.036 & N.S. & 0.166 & $<0.05$ & 0.029 & N.S. & 0.250 & $<0.001$ \\
\hline Weight (kg) & 0.605 & $<0.001$ & 0.266 & $<0.001$ & 0.679 & $<0.001$ & 0.277 & $<0.001$ \\
\hline BMI $\left(\mathrm{kg} / \mathrm{m}^{2}\right)$ & 0.889 & $<0.001$ & 0.211 & $<0.01$ & 0.870 & $<0.001$ & 0.113 & $<0.01$ \\
\hline$W C(\mathrm{~cm})$ & 0.499 & $<0.001$ & 0.162 & $<0.05$ & 0.477 & $<0.001$ & 0.092 & $<0.05$ \\
\hline Handgrip strength (kg) & 0.287 & $<0.001$ & 0.188 & $<0.01$ & 0.278 & $<0.001$ & 0.167 & $<0.001$ \\
\hline Whole-body BMD $\left(\mathrm{g} / \mathrm{cm}^{2}\right)$ & 0.306 & $<0.001$ & -0.026 & N.S. & 0.217 & $<0.001$ & 0.028 & N.S. \\
\hline Arm BMD $\left(\mathrm{g} / \mathrm{cm}^{2}\right)$ & 0.366 & $<0.001$ & 0.041 & N.S. & 0.282 & $<0.001$ & 0.171 & $<0.001$ \\
\hline Lumbar spine BMD $\left(\mathrm{g} / \mathrm{cm}^{2}\right)$ & 0.150 & $<0.05$ & -0.137 & $<0.05$ & 0.206 & $<0.001$ & -0.018 & N.S. \\
\hline Leg BMD $\left(\mathrm{g} / \mathrm{cm}^{2}\right)$ & 0.414 & $<0.001$ & 0.082 & N.S. & 0.360 & $<0.001$ & 0.203 & $<0.001$ \\
\hline HbA1c (\%) & -0.040 & N.S. & -0.031 & N.S. & 0.056 & N.S. & 0.000 & N.S. \\
\hline $\mathrm{TG}(\mathrm{mg} / \mathrm{dl})$ & -0.033 & N.S. & 0.012 & N.S. & 0.031 & N.S. & -0.003 & N.S. \\
\hline $\mathrm{HDL}-\mathrm{C}(\mathrm{mg} / \mathrm{dl})$ & -0.003 & N.S. & -0.114 & N.S. & -0.093 & $<0.05$ & 0.036 & N.S. \\
\hline TG/HDL ratio & -0.018 & N.S. & 0.060 & N.S. & 0.046 & N.S. & -0.024 & N.S. \\
\hline $\mathrm{SBP}(\mathrm{mmHg})$ & 0.068 & N.S. & -0.069 & N.S. & 0.077 & $<0.05$ & -0.057 & N.S. \\
\hline $\mathrm{DBP}(\mathrm{mmHg})$ & 0.157 & $<0.05$ & 0.007 & N.S. & 0.073 & $<0.05$ & -0.087 & $<0.05$ \\
\hline baPWV (cm/s) & -0.240 & $<0.001$ & -0.241 & $<0.001$ & -0.133 & $<0.001$ & -0.152 & $<0.001$ \\
\hline
\end{tabular}

ASM appendicular skeletal muscle mass, $H t$ height, $W t$ weight, $B M I$ body mass index, $W C$ waist circumference, $B M D$ bone mineral density, $H b A 1 c$ glycohemoglobin A1c, $T G$ triglycerides, HDL-C high-density lipoprotein cholesterol, SBP systolic blood pressure, DBP diastolic blood pressure, baPWV brachial-ankle pulse wave velocity

subjects were not significantly different. When sarcopenia was defined by weight-adjusted ASM, baPWV in class 1 and 2 sarcopenic subjects was significantly higher than that in normal subjects $(P<0.05)$, but when defining sarcopenia by height-adjusted ASM, that was not significantly different.

Health-related indexes in middle-aged and elderly women are shown in Table 5. In height-adjusted definition, BMI, WC, and handgrip strength in class 1 and 2 sarcopenic subjects were significantly lower than those in normal subjects $(P<0.05)$, but not in weightadjusted definition. When sarcopenia was defined by height-adjusted ASM, whole-body BMD, arm BMD, lumbar spine BMD, and leg BMD in class 1 and class 2 sarcopenic subjects were significantly lower than those in normal subjects $(P<0.05)$, but when sarcopenia was defined by weight-adjusted ASM, those were not significantly different. In definition of ASM/Wt, TG, TG/HDL-C, SBP, and DBP in class 1 and class 2 sarcopenic subjects were significantly higher than those in normal subjects $(P<0.05)$, but in definition of $\mathrm{ASM} / \mathrm{Ht}^{2}$, those were not significantly different. In both definitions, baPWV in class 1 and 2 sarcopenic subjects was significantly higher than that in normal subjects $(P<0.05)$.

\section{Discussion}

The major findings of our study were that heightadjusted ASM showed stronger associations with risk factors for osteoporosis than weight-adjusted ASM, and conversely, weight-adjusted ASM was more strongly associated with risk factors for cardiometabolic diseases than height-adjusted ASM.

Kim et al. previously proposed cut-off values for class 1 and class 2 sarcopenia in Korean people [15] of 32.2 and $29.1 \%$ for men and 25.6 and $23.0 \%$ for women, respectively, using the weight-adjusted definition. They also reported prevalence rates of class 1 and class 2 sarcopenia of 29.5 and $9.7 \%$ for elderly men and 30.3 and $11.8 \%$ for elderly women (age, $\geq 65$ years), respectively, using the same definition. In this study, the prevalence rates of sarcopenia were higher than those reported in the Korean study for both sexes and our cutoff values were considerably higher because weight, $\mathrm{BMI}$, and WC in our young group were lower than those in the young group from the Korean study. Moreover, absolute muscle mass in our young group was relatively higher than that reported in the Korean study.

Sanada et al. previously reported that BMI and WC in sarcopenic subjects (defined by height-adjusted ASM) were significantly lower than those in normal subjects 
Table 4 Characteristics of middle-aged and elderly men with sarcopenia defined by height-adjusted ASM or weight-adjusted ASM

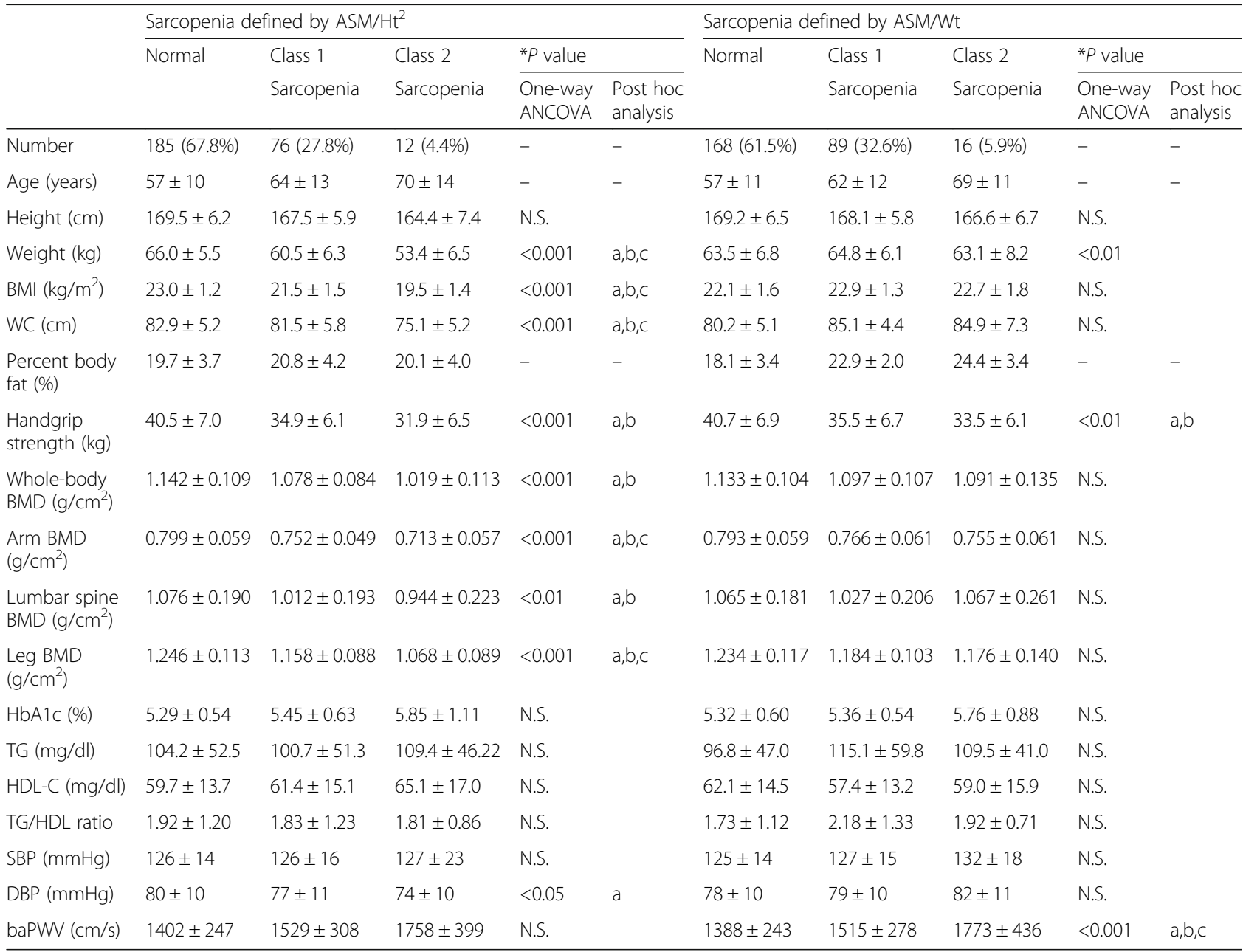

Data are presented as mean $\pm S D$

$B M I$ body mass index, WC waist circumference, BMD bone mineral density, HbA1C glycohemoglobin A1c, TG triglycerides, HDL-C high-density lipoprotein cholesterol, $S B P$ systolic blood pressure, $D B P$ diastolic blood pressure, baPWV brachial-ankle pulse wave velocity

*One-way ANCOVA adjusted for age and percent body fat and post hoc analysis using the least significant difference $t$ test (mean difference between two groups): a, Normal vs. class $1 ;$ b, Normal vs. class $2 ; c$, class 1 vs. class $2 ;$ all $P<0.05$

[14]. Abe et al. also reported that, after adjusting for age, BMI, WC, and percent body fat were lower when using the height-adjusted definition [16]. This suggests that sarcopenia defined by height-adjusted ASM does not detect people at higher risk of obesity. However, Janssen et al. found that sarcopenia defined by weight-adjusted ASM was associated with higher BMI in both men and women [7]. In the present study, when sarcopenia was defined by height-adjusted ASM, WC in sarcopenic subjects was significantly lower than that in normal subjects $(P<0.05)$, but when defined by weight-adjusted ASM, WC was not significantly different for both men (Table 4) and women (Table 5). Moreover, Wt, BMI, and WC were positively correlated with height-adjusted ASM, but negatively correlated with weight-adjusted ASM after adjusting for age in both men and women (Table 2). These results suggest that sarcopenia defined by weight- adjusted ASM is associated with abdominal obesity. Thus, defining sarcopenia by weight-adjusted ASM can better detect sarcopenic individuals at higher risk of obesity than when defining sarcopenia by heightadjusted ASM.

Recently, Hamasaki et al. reported that the ratio of lower extremity muscle mass to weight measured by bioelectrical impedance was negatively correlated with BMI, WC, body fat mass, percent body fat, subcutaneous fat area, and serum-free fatty acid concentration [17]. In this study, after adjusting age, ASM/Wt was more strongly correlated with risk factors for cardiometabolic diseases than ASM/ $\mathrm{Ht}^{2}$ (Table 2). For example, ASM/ Wt was negatively correlated with TG and positively correlated with $\mathrm{HDL}-\mathrm{C}$, but $\mathrm{ASM} / \mathrm{Ht}^{2}$ was not correlated with them in both men and women. ASM/Wt was also strongly correlated with percent body fat (men; 
Table 5 Characteristics of middle-aged and elderly women with sarcopenia defined by height-adjusted ASM or weight-adjusted ASM

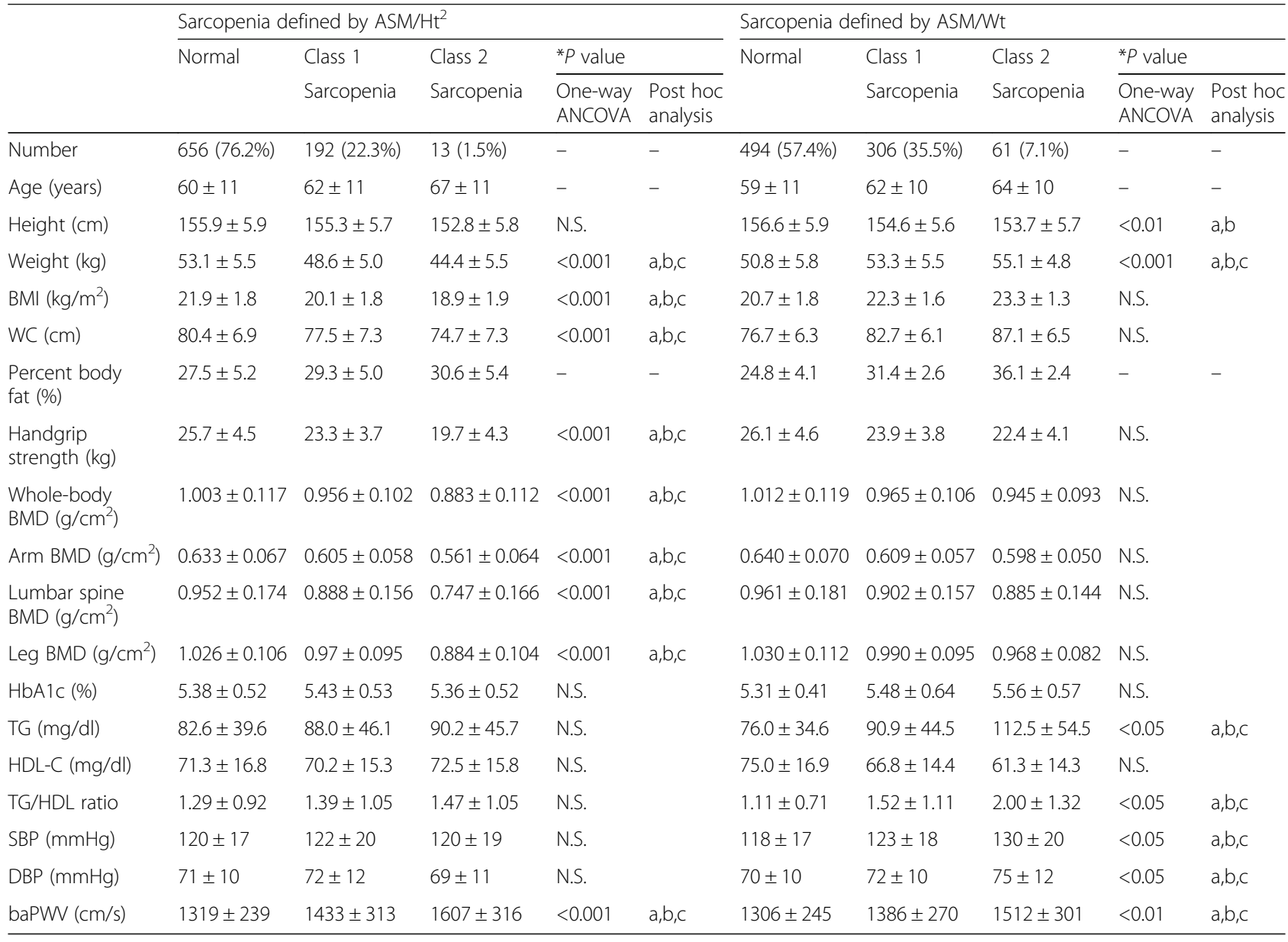

Data are presented as mean \pm SD

$B M I$ body mass index, WC waist circumference, $B M D$ bone mineral density, $H b A 1 c$ glycohemoglobin A1c, $T G$ triglycerides, $H D L-C$ high-density lipoprotein cholesterol, SBP systolic blood pressure, DBP diastolic blood pressure, baPWV brachial-ankle pulse wave velocity

*One-way ANCOVA adjusted for age and percent body fat and post hoc analysis using the least significant difference $t$ test (mean difference between two groups): a, Normal vs. class $1 ; \mathrm{b}$, Normal vs. class $2 ; c$, class 1 vs. class 2 ; all $P<0.05$

$R=-0.73$, women; $R=-0.90)$. Thus, it is considered that the associations between ASM/Wt and risk factors for cardiometabolic diseases were caused by negative correlations between ASM/Wt and percent body fat. After adjusting age and percent body fat, most of the significant differences were disappeared (Table 3). Therefore, it was shown that percent body fat contributed strongly to the associations between ASM/Wt and risk factors for cardiometabolic diseases. However, even after adjusting age and percent body fat, sarcopenia defined by weight-adjusted ASM in women was significantly associated with risk factors for cardiometabolic diseases, including TG, TG/ HDL-C, SBP, and DBP (Table 5). On the other hand, sarcopenia defined by height-adjusted ASM was not significantly associated with them. For men, in definition of ASM/Wt, baPWV in sarcopenic subjects was significantly higher than that in normal subjects, but in definition of $\mathrm{ASM} / \mathrm{Ht}^{2}$, baPWV was not significantly different (Table 4).
These results suggested that even after adjusting percent body fat, sarcopenia defined by weight-adjusted ASM can detect sarcopenic individuals at higher risk of cardiometabolic diseases with more sensitivity than when defined by height-adjusted ASM.

With regard to risk factors for osteoporosis, a previous study reported that sarcopenia defined by height-adjusted ASM was associated with lower BMD in both men and women [14]. In contrast, another study reported that osteoporosis was not significantly associated with muscle mass/weight [12]. In the present study, for both men and women, whole-body BMD, arm BMD, lumbar spine BMD, and leg BMD in sarcopenic subjects (defined by heightadjusted ASM) were significantly lower than those for normal subjects. When defined by weight-adjusted ASM, however, those in sarcopenic subjects were not significantly different (Tables 4 and 5 ). These results suggest that sarcopenia defined by height-adjusted ASM can better 
detect sarcopenic individuals at higher risk of osteoporosis than when defined by weight-adjusted ASM. However, this study measured whole-body and regional BMD using only whole-body DXA. Lumbar spine BMD should be examined by the specific manner. This point is a limitation in the present study.

\section{Conclusions}

In practice, the use of height-adjusted ASM has the advantage over the weight-adjusted ASM to detect risk factors for osteoporosis. Conversely, the use of weight-adjusted ASM has the advantage to detect risk factors for cardiometabolic diseases.

\section{Abbreviations}

baPWV: Brachial-ankle pulse wave velocity; BMD: Bone mineral density; BMI: Body mass index; CVD: Cardiovascular diseases; DBP: Diastolic blood pressure; DXA: Dual-energy X-ray absorptiometry; HbA1c: Glycohemoglobin A1C; HDL-C: High-density lipoprotein cholesterol; Ht: Height; SBP: Systolic blood pressure; SD: Standard deviations; TG: Triglycerides; WC: Waist circumference; Wt: Body weight

\section{Acknowledgements}

We thank the subjects who participated in this study.

\section{Funding}

This work was supported by a Grant-in-Aid for Scientific Research from the Ministry of Education, Culture, Sports, Science and Technology of Japan (\#24300239, K. Sanada).

\section{Availability of data and materials}

The datasets during the current study are available from the corresponding author on reasonable request.

\section{Authors' contributions}

TF contributed to study design, analyzed the data, and drafted the manuscript; MM was an administrator of NEXIS cohort and made critical revisions to the manuscript; KS supervised the study, contributed to study design, and made critical revisions to the manuscript. MI, HM, HK, YG, and RK measured fitness and metabolic risk parameters. All authors read and approved the final manuscript.

\section{Competing interests}

The authors declare that they have no competing interests.

\section{Consent for publication}

Not applicable.

\section{Ethics approval and consent to participate}

All subjects gave their written informed consent before enrolling in the study. The study was performed in accordance with the guidelines of the Declaration of Helsinki and was approved by the Human Research Committee of the National Institute of Health and Nutrition, Tokyo, Japan.

\section{Author details}

${ }^{1}$ College of Sport and Health Science, Ritsumeikan University, 1-1-1 Noji Higashi, Kusatsu, Shiga 525-8577, Japan. ${ }^{2}$ Department of Health Promotion and Exercise, National Institute of Health and Nutrition, 1-23-1 Toyama, Shinjuku-ku, Tokyo 162-8636, Japan. ${ }^{3}$ Faculty of Letters, Kokushikan University, 4-28-1, Setagaya, Setagaya-ku, Tokyo 154-8515, Japan. ${ }^{4}$ Faculty of Sport Sciences, Waseda University, 2-579-15, Mikajima, Tokorozawa, Saitama 359-1192, Japan
Received: 1 November 2016 Accepted: 6 February 2017

Published online: 13 February 2017

\section{References}

1. Rosenberg $\mathbb{I H}$. Sarcopenia: origins and clinical relevance. J Nutr. 1997; 127:990S-1S.

2. Baumgartner RN, Koehler KM, Gallagher D, Romero L, Heymsfield SB, Ross $\mathrm{RR}$, et al. Epidemiology of sarcopenia among the elderly in New Mexico. Am J Epidemiol. 1998:147:755-63.

3. Tanimoto $Y$, Watanabe M, Sun W, Tanimoto K, Shishikura K, Sugiura Y, et al. Association of sarcopenia with functional decline in community-dwelling elderly subjects in Japan. Geriatr Gerontol Int. 2013;13:958-63.

4. Verschueren S, Gielen E, O'Neill TW, Pye SR, Adams JE, Ward KA, et al. Sarcopenia and its relationship with bone mineral density in middle-aged and elderly European men. Osteoporos Int. 2013;24:87-98.

5. Gale CR, Martyn CN, Cooper C, Sayer AA. Grip strength, body composition, and mortality. Int J Epidemiol. 2007:36:228-35.

6. Sayer AA, Dennison EM, Syddall HE, Gilbody HJ, Phillips DI, Cooper C. Type 2 diabetes, muscle strength, and impaired physical function: the tip of the iceberg? Diabetes Care. 2005;28:2541-2.

7. Janssen I, Heymsfield SB, Ross R. Low relative skeletal muscle mass (sarcopenia) in older persons is associated with functional impairment and physical disability. J Am Geriatr Soc. 2002;50:889-96.

8. Hong W, Cheng Q, Zhu X, Zhu H, Li H, Zhang X, et al. Prevalence of sarcopenia and its relationship with sites of fragility fractures in elderly Chinese Men and women. PLoS One. 2015:10:e0138102.

9. Kim S, Won CW, Kim BS, Choi HR, Moon MY. The association between the low muscle mass and osteoporosis in elderly Korean people. J Korean Med Sci. 2014;29:995-1000

10. Park S, Ham JO, Lee BK. A positive association between stroke risk and sarcopenia in men aged $>/=50$ years, but not women: results from the Korean National Health and Nutrition Examination Survey 2008-2010. J Nutr Health Aging. 2014:18:806-12.

11. Byeon CH, Kang KY, Kang SH, Bae EJ. Sarcopenia is associated with Framingham risk score in the Korean population: Korean National Health and Nutrition Examination Survey (KNHANES) 2010-2011. J Geriatr Cardiol. 2015;12:366-72.

12. Pongchaiyakul C, Limpawattana $P$, Kotruchin $P$, Rajatanavin R. Prevalence of sarcopenia and associated factors among Thai population. J Bone Miner Metab. 2013;31:346-50.

13. Lim S, Kim JH, Yoon JW, Kang SM, Choi SH, Park YJ, et al. Sarcopenic obesity: prevalence and association with metabolic syndrome in the Korean Longitudinal Study on Health and Aging (KLoSHA). Diabetes Care. 2010;33:1652-4.

14. Sanada K, Miyachi M, Tanimoto M, Yamamoto K, Murakami H, Okumura S, et al. A cross-sectional study of sarcopenia in Japanese men and women: reference values and association with cardiovascular risk factors. Eur J Appl Physiol. 2010;110:57-65.

15. Kim YS, Lee Y, Chung YS, Lee DJ, Joo NS, Hong D, et al. Prevalence of sarcopenia and sarcopenic obesity in the Korean population based on the Fourth Korean National Health and Nutritional Examination Surveys. J Gerontol A Biol Sci Med Sci. 2012;67:1107-13.

16. Abe T, Thiebaud RS, Loenneke JP, Bemben MG, Loftin M, Fukunaga T. Influence of severe sarcopenia on cardiovascular risk factors in nonobese men. Metab Syndr Relat Disord. 2012;10:407-12.

17. Hamasaki H, Kawashima $Y$, Adachi H, Moriyama S, Katsuyama H, Sako A, et al. Associations between lower extremity muscle mass and metabolic parameters related to obesity in Japanese obese patients with type 2 diabetes. PeerJ. 2015;3:e942. 